\title{
COLLAPSE OF SEMIFLEXIBLE POLYMERS IN TWO DIMENSIONS. MONTE CARLO SIMULATIONS
}

\author{
A. KolińsKi, M. Vieth and A. SiKorSKi \\ Department of Chemistry, University of Warsaw, Pasteura 1, 02-093 Warszawa, Poland \\ (Received July 18, 1990).
}

\begin{abstract}
We present Monte Carlo simulations of a two-dimensional square lattice semiflexible polymer model. Short-range repulsive potential (excluded volume) and long-range attractive (segment-segment) potential are included. Particular attention was paid to a phase transition of a model polymer chain from a random coil state to a dense globule. The transition temperature increases with the chain length and decreases with the stifness of the model chain. The cooperativity of the transition increases with the polymer stiffness.
\end{abstract}

PACS numbers: 02.60.+y, 82.35. $+\mathrm{t}$

\section{Introduction}

A collapse transition of chain polymers is a long-standing problem of theoretical and computational physics [1-3]. The collapse occurs when an attractive component of a potential of the mean force of intra-polymer interactions (in a solution) overrides short rangc excluded volume repulsions between polymer segments. The problem is important for many reasons. It is strictly related to the theory of critical phenomena [2-4], and it is often discussed in the context of the coil-globule transition in globular proteins [5-7]. Several theoretical and computational works (predominantly Monte Carlo simulations) concerned the case of long flexible polymers models [8-13]. Much less effort has been focused on very important questions of interplay between short range interactions i.e., conformational stiffness of the polymer and long range mean-force interactions of polymer segments [14]. The term "long-range interactions" in polymer physics means interactions between polymer units (monomers) which are separated by long distance down the chain backbone but are not necessary distant in space occupied by polymer. Actually 
these long-range interactions are usually of van der Waals type and consequently effective on rather limited distance, comparable to the size of a single monomer. Even mean-field approximate considerations have shown that the character of the polymer collapse changes dramatically, when one takes into consideration the effect of short range stiffness of the chain. Even stronger effect of relatively small conformational stiffness on the chain collapse has been demonstrated in recent Monte Carlo studies of three-dimensional lattice polymers [14]. It has been shown that this kind of models can explain many aspects of the mechanism of the globular protein folding process [5-7].

In this context it seems to be interesting to examine a similar effect in the case of two dimensional polymers [15-17]. On the experimental level the collapse of two dimensional polymers can be realized on a strongly absorbing surface or in a thin film of a solution [18-19]. There are some computer simulation works on the polymer chain collapse in two dimensions [20-24]. The case of a flexible square lattice model polymers has been studied by Baumgartner [21], ant the present results can be easily compared with these studies. It should be noted that two dimensional systems may exhibit even more pronounced effect of chain stifness than it has been seen in three dimensional case.

The meaning of chain stiffness or, more precisely, the ratio of conformational stiffness to the long range attractive force, in the case of two-dimensions, needs some additional comment. In the simplest case the interpretation is the same as for the three dimensional systems. Ilowever it should be noted that in the 2nd case this ratio can be in fact moderaled by the strength of the absorbing force of the surface. In the present work we use the simplest possible lattice representation of the polymer chain, which occupies $n$ consecutive points in a simple square lattice. The local stiffness is accounted by energetic preference for collinear orientation of two consecutive "bonds" joining the lattice points (polymer beads). The repulsive part of the long range interactions is simulated by the requirement of a single occupancy of any lattice points. The long-range attractive force extends to the distance of a single lattice spacing, i.e. acts between the nearest (nonbonded) neighbours on the lattice. It should be pointed out that it is treated as a potential of the mean force. Thus the model is quite general and can be scaled onto wide class of related physical systems.

\section{Model and Monte Carlo procedure}

The model macromolecule consist of $n-1$ bonds (polymer segments) joining $n$ lattice points. Thus the chain backbone may be represented as an ordered set of vectors of the type $l=[ \pm 1,0]$ and $[0, \pm 1]$. Long-range interactions are described by the following potential of the mean force:

$$
V_{i j}= \begin{cases}\infty, & r_{i j}=0 \\ -\varepsilon_{a}, & r_{i j}=|l|=1 \\ 0, & r_{i j}>1\end{cases}
$$

- with $|i-j|>1$, where $r_{i j}$ is a distance between $i$-th and $j$-th polymer beads 
counted down the chain. Short-range interactions are described by the potential:

$$
U_{i}= \begin{cases}-\varepsilon_{g} & l_{i}=l_{i+1} \\ 0, & \text { otherwise }\end{cases}
$$

where $l_{i}$ is a vector from $i$-th to $(i+1)$-th bead, and $i=1,2, \ldots, n-1$.

The total conformational energy for a given state of the model chain can be calculated as a sum of the both contributions:

$$
E=\sum_{i=1}^{n} \sum_{j=i+2}^{n} V_{i j}+\sum_{i=1}^{n-1} U_{i} .
$$

The Metropolis scheme is built-in into the Monte Carlo sampling algorithm [1, 22]. Consequently the transition probability from one state (old) to another (new) is defined as:

$$
P_{\text {old } \rightarrow \text { new }}= \begin{cases}1, & E_{\text {new }} \leq E_{\text {old }} \\ \exp \left(-\Delta E / k_{\mathrm{B}} T\right), & E_{\text {new }}>E_{\text {old }}\end{cases}
$$

where $\Delta E=E_{\text {new }}-E_{\text {old }}, k_{\mathrm{B}}$ is the Boltzmann's constant and $T-$ temperature. The reduced temperature of the model system $T^{*}$ can be defined basing on value of $\varepsilon_{a}$ or $\varepsilon_{g}$. The first possibility was chosen because of the easy reference to other works where $\varepsilon_{g}=0$. Thus $T^{*}=k_{\mathrm{B}} T / \varepsilon_{a}$.

The method of generation of trial transitions from a given "old" state to a "new" one requires detailed discussion, since it is crucial for the ergodicity of the algorithm. It should be noted that most of the algorithms used in the past are in fact nonergodic. It can be shown that the lack of ergodicity is not too important for the three-dimensional polymer models (subclasses of the ergodicity are wide enough to give correct estimations of the system properties). In the case of two dimensions lack of ergodicity may be much more dangerous [26-27].

The following set of conformational transitions was used in the Monte Carlo algorithm in the present work:

1. Two bond permutation (Fig.1a).

2. Thrce bond "crankshaft" motion (Fig:1b).

3. End bonds (one and two) random rearrangements (Fig.1c).

4. "Wave" motions (Fig.1d) of randomly chosen piece of the chain.

In a single cycle (an internal time unit of the algorithm) $(n-2)$ attempts of the first type, $(n-3)$ attempts of the second type, 2 attempts of end motions and one attempt of the "wave" motion were performed. The last type of micromodification is essential for the ergodicity of the process [5-7]. Since the microscopic reversibility and the detailed balance of any type of motion described above is obvious, one need only to show that each conformation of the chain can be reached in the finite number of steps. Consider for example a completely folded (close packed) 
a
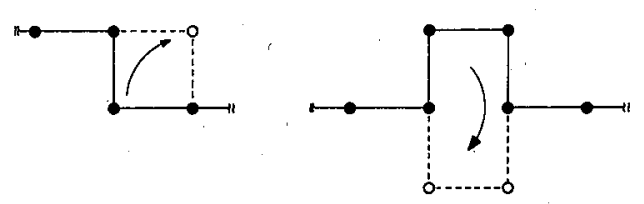

c
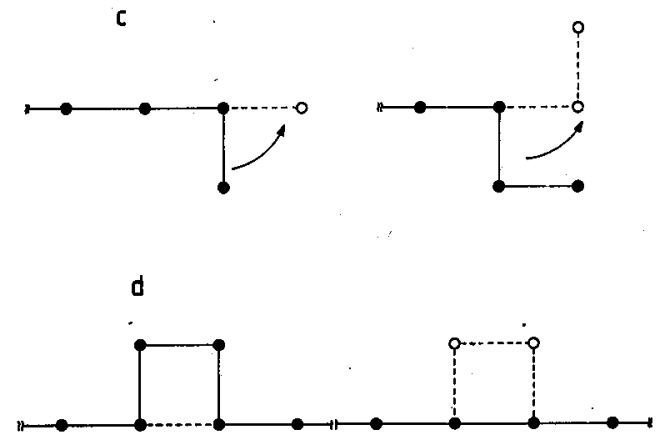

Fig. 1. Set of micromodifications used in the Monte Carlo sampling algorithm.

conformation with chain ends buried within such a globular state. The set of micromodifications $1-3$ is usually unable to unfold such a configuration [26-27]. The same is true for the "reptation" algorithm used in several works. "Wave" motion clearly allows the folding and the unfolding of every close packed configurations. Consequently our algorithm is ergodic since the last type of motion ensures the stationary condition. The sequence of attempted motions and the choice of a piece of the chain treated at the moment are governed by a pseudorandom mechanism.

The strategy of sampling was the following. First, the randomly coiled chain was gencrated by a separate algorithm. Then the coil-globule transition temperature was approximately localized in a several relatively short MC simulation runs. This allowed for the appropriate choice of temperature increments for a series of production runs. A final production run consisted of at least one cooling-heating sequence. Every sampling run was preceded by an equilibration run for every temperature. At each temperature sampling runs consisted of up to the order of $10^{6}$ cycles of the Monte Carlo algorithm described above in the case of the longest chain $(n=300)$. We remind that a simple cycle obeys many micromodifications of various types. Since we do not notice any tendency to a hysteresis of the measured properties near the collapse transition (during a heating-cooling route) the reported data represent values averaged over the all sampling runs at a given temperature. Various properties of the model chain (e.g. the mean-square radius of gyration) werc measured every 100 cycles of the $\mathrm{MC}$ algorithm. Due to the Metropolis sampling procedure the average values are calculated as a simple arithmetical averages. The accuracy of the estimation of various properties depends on 
the property under consideration and can be evaluated from the dispersion of the results of several independent runs, i.e. of runs for the same temperature, the same chain length and the same ratio $\varepsilon_{g} / \varepsilon_{a}$ but started from a different initial state and using a different sequence of pseudorandom numbers. All the computations were performed on UNOX/AT-386 computer using an extremely effective algorithm. The algorithm uses the direct reference list for storing chain conformation. Thus the test of a double occupancy for a given bead requires a single reference to the occupancy list and the computation of a Boltzmann factor in the Metropolis scheme requires just few arithmetical operations since exponential factors are computed once at the very beginning of the simulation and stored in an appropriate array. All the above makes computations feasible on a fast personal computer.

\section{Results and discussion}

Simulations were performed for several values of chain length and four values of the stiffness parameter $\varepsilon_{g} / \varepsilon_{a}$. Since the case of $\varepsilon_{g} / \varepsilon_{a}=0$ has been already examined the majority of our computations concerned semiflexible polymers i.e. $\varepsilon_{g} / \varepsilon_{a}=1, \varepsilon_{g} / \varepsilon_{a}=2$ and $\varepsilon_{g} / \varepsilon_{a}=4$. It allows us for the elucidation of the effect of the chain stiffness on the collapse transition of a linear macromolecule.

The model systems are characterized by the average conformational energy. $\langle E\rangle$, the average heat capacity computed from energy fluctuations $C_{V} / k_{\mathrm{B}}=$ $D^{2}(E)=\left(\left\langle E^{2}\right\rangle-\langle E\rangle^{2}\right)$, the average size of the polymer coil (globule) measured by the mean-square radius of gyration $\left\langle S^{2}\right\rangle$ which is defined as usual [10]:

$$
\left\langle S^{2}\right\rangle=\left\langle n^{-1} \sum_{i=1}^{n}\left(r_{i}-r_{\mathrm{CM}}\right)^{2}\left(r_{i}-r_{\mathrm{CM}}\right)^{2}\right\rangle .
$$

where $r_{i}$ and $r_{\mathrm{CM}}$ are the coordinates of $i$-th polymer bead and center of mass of the coil respectively. In all cases $\langle.$.$\rangle means ensemble averages estimated over a$ $M C$ run. Additional various a verage parameters describing the arrangement of the chain segments within a coil (globule) are also computed. The most useful seems to be the average length of a sequence of collinear bonds $\left\langle n_{c}\right\rangle$ [14]. This parameter corrcsponds to the local ordering within the chain. Global ordering of a finite chain may be described by the normalized fraction $f_{x y}$ of the chain bonds oriented in parallel or (antiparallel) manner down the leading direction of the chain vectors ( $x$ or $y$ axis). Suppose that within a given conformation $n_{x}$ bonds are orientated down the $x$ axis $\left(n_{x}+n_{y}=n-1\right)$. For each conformation examined one may compute $f_{x y}$ as follows

$$
f_{x y}=\left\{\begin{array}{lll}
n_{x} /(n-1) & \text { for } & n_{x}>n_{y} \\
n_{y} /(n-1) & \text { for } & n_{y}>n_{x}
\end{array}\right.
$$

The order parameter $p$ :

$$
p=2\left\langle f_{x y}\right\rangle-1
$$




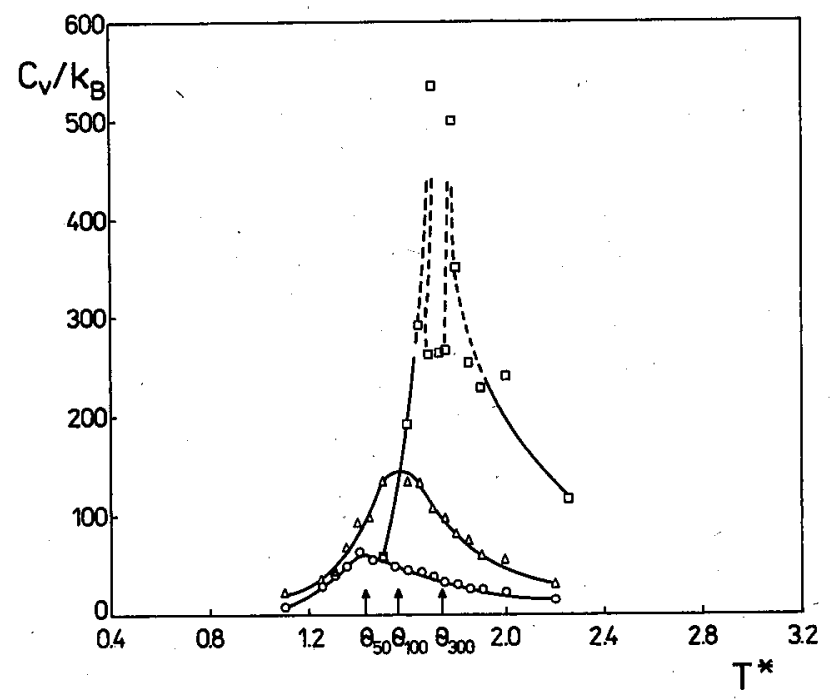

Fig. 2. Plot of the reduced heat capacity $C_{V} / k_{\mathrm{B}}$ vs. reduced temperature $T^{*}$ for various chain length: $n=50$ (open circles), $n=100$ (open triangles) and $n=300$ (open squares). The case of $\varepsilon_{g} / \varepsilon_{a}=2$.

reflects the orientational ordering of the entire chain. For a long flexible, disordered coil, or a globule, $p \approx 0$. For a short stiff chain one may expect $p \approx 1$.

At first we illustrate the method of the localization coil-globule (collapse) transition temperature for the chosen value of stiffness parameter $\varepsilon_{g} / \varepsilon_{a}=2$. In the Fig. 2 the reduced heat capacity $C_{V} / k_{\mathrm{B}}=D^{2}\left(E / k_{\mathrm{B}} T\right)$ is plotted versus the reduced temperature $T^{*}=k_{\mathrm{B}} T / \varepsilon_{a}$ for various values of chain length. With increasing chain length $T_{t}^{*}$ (at the maximum of the $C_{V} / k_{\mathrm{B}}$ ) monoticaly increases. The maximum of $C_{V}$ versus $T^{*}$ plots may be identified with the collapse transition of a polymer coil. For shorter chains there is a single maximum. For the longest chain under consideration $(n=300)$ there are two clear peaks on the $C_{V} / k_{\mathrm{B}}$ plot both observed during the slow heating as well as during the slow cooling of the system. Although we can not exclude some marginal possibility of the quenching of the system it is very likely that these two peaks are not artifacts but correspond to two separate processes of an internal rearrangement of the globule at the collapse temperature. A finite size scaling, which uses the expected critical exponents for the infinite system may be used for extrapolation of transition temperature $T_{t}^{*}(n)$ to the case of an the infinite chain length $T_{t}^{*}(\infty)$ [21]. Other temperature dependent characteristics of the model system can be used for the evaluation of $T_{t}^{*}(n)$. In the Fig. 3(a-d) the average reduced conformational energy $\left\langle E / k_{\mathrm{B}} T\right\rangle$, the average square radius of gyration $\left\langle S^{2}\right\rangle$, the average length $\left\langle n_{c}\right\rangle$ of collinear. sequence of the model chain bonds and the reduced heat capacity $C_{V} / k_{\mathrm{B}}$ are plotted versus the reduced temperature $T^{*}$ for the case of $n=100\left(\varepsilon_{g} / \varepsilon_{a}=2\right.$ and 
$\left.\varepsilon_{g} / \varepsilon_{a}=4\right)$. It is clear that there are well defined change of the system properties at $T_{t}^{*}$. Since the energy curve seems to be continuous, the system does not exhibit existence of metastable states and there is rather not possible to see any hysteresis of the result, one should expect that the transition is of the second-order in the limit of $n \longrightarrow \infty$. For finite systems the transition is also continuous (however cooperatively of the crossover is rather high) in contrast to the similar semiflexible systems in three dimensions [14].

The cooperativity of the collapse transition mentioned above increases with the increasing of the chain stiffness. In the case of semiflexible polymers $\left(\varepsilon_{g} / \varepsilon_{a}>0\right)$ one may notice very sharp increase of the order parameter $\left\langle n_{c}\right\rangle$ at the transition region. It means that collapse induced an excess stiffness of the chain, while flexible polymers do not exhibit any observable change of conformational stiffness at the transition. This is illustrated in the Fig. $3(\mathrm{c}-\mathrm{d})$. Even more explicitly the effect may be abserved in series of snapshots of configurations of model systems. In Fig. 4 (a-d) representative conformations of a high temperature coil state and low temperature globular state are plotted. In the Table I we present the comparison of the numerical values of global order parameters $p$ at the globular state for various values of $\varepsilon_{g} / \varepsilon_{a}$ and chain lengths. There is a significant level of globular ordering for stiff, and not too long polymers.

\section{TABLE I}

The comparison of the numerical

values of global order parameters $p$

of the globular state for various

values of $\varepsilon_{g} / \varepsilon_{a}$ and chain lengths.

\begin{tabular}{r|c|l|c}
\hline \multicolumn{1}{c}{$n$} & $\varepsilon_{g} / \varepsilon_{a}$ & \multicolumn{1}{c}{$T^{*}$} & $p$ \\
\hline 50 & 2 & 1.1 & 0.6289 \\
100 & 0 & 0.6 & 0.0914 \\
100 & 1 & 0.8 & 0.2957 \\
100 & 2 & 1.25 & 0.7248 \\
100 & 4 & 1.3 & 0.9368 \\
300 & 1 & 1.0 & 0.2497 \\
300 & 2 & 1.5 & 0.7322
\end{tabular}

The ratio of the local conformational stiffness to the long-range interactions ratio changes not only the character of the collapse transition but also shifts the transition temperature. It can be seen in the Fig. 5. Table II contains numerical values of $T_{i}^{*}(n)$ for various systems under consideration and when possible also $T_{t}^{*}(\infty)$ are also given. $T_{t}^{*}(\infty)$ values may be obtained by the extrapolation of $T_{t}^{*}$ to $n=\infty$, assuming that $T_{t}^{*}(\infty)-T_{t}^{*}(n)$ scales as $n^{-\varphi t}$ with $\varphi t \approx 0.64$. In spite of data scattering there is a clear dependence of $T_{t}^{*}(n)$ on chain stiffness $\varepsilon_{g} / \varepsilon_{a}$ (for a given finite $n$ as well as for the limit of $n=\infty$ value). Namely $T_{t}^{*}(n)$ increases with chain length and with the value of $\varepsilon_{g} / \varepsilon_{a}$. 
a

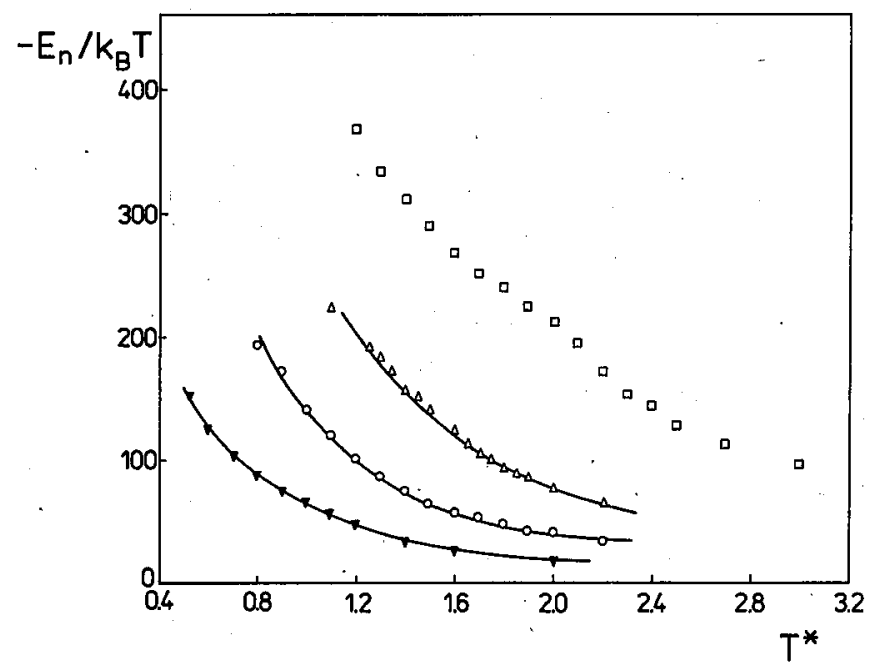

b

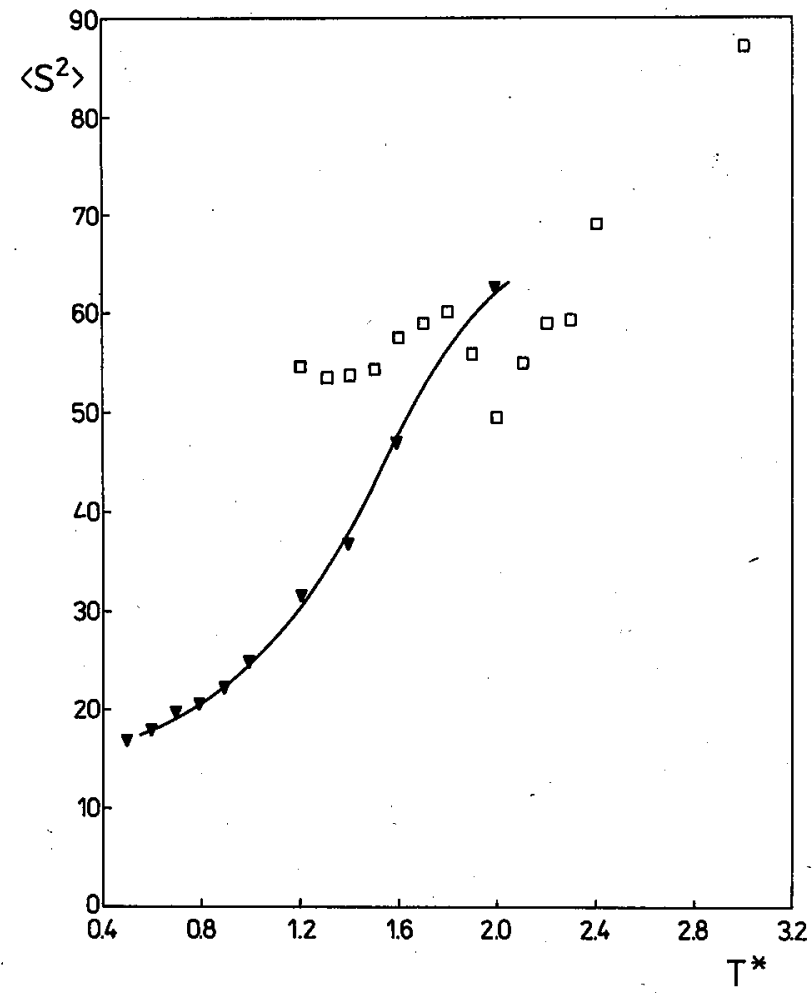

Fig. 3(a,b). The average reduced conformational energy $E / k_{\mathrm{B}} T(\mathrm{a})$, the mean-square radius of gyration $\left\langle S^{2}\right\rangle(\mathbf{b})$. 


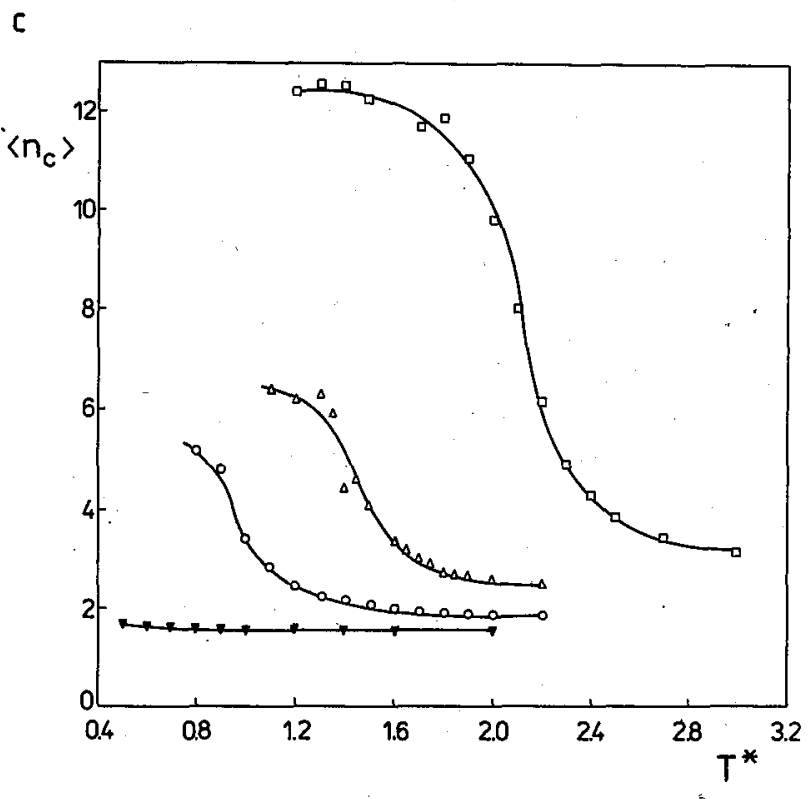

d

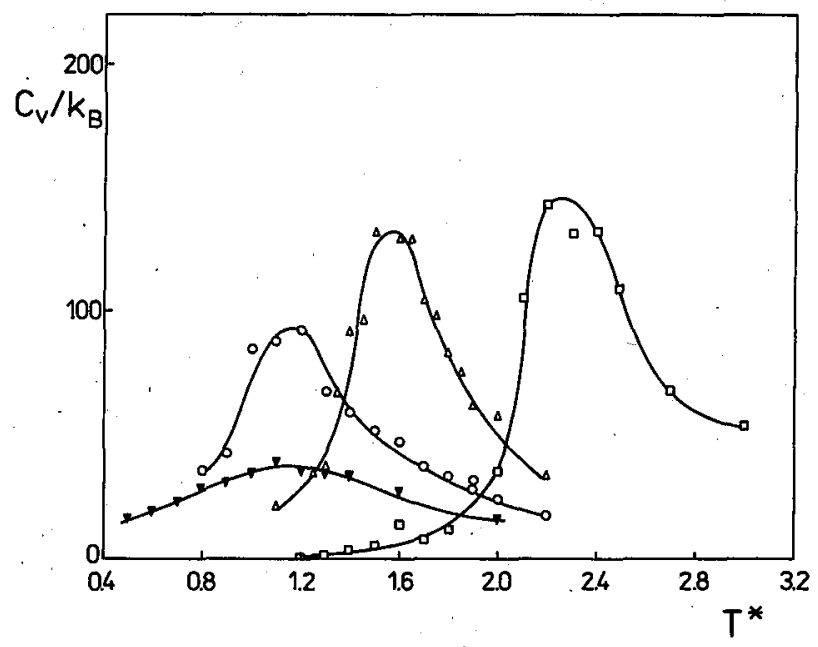

Fig. 3(c,d). The average collinear bond sequence $\left\langle n_{c}\right\rangle(\mathrm{c})$, the reduced heat capacity $C_{V} / k_{\mathrm{B}}(\mathrm{d})$ versus reduced temperature $T^{*}$. Cases $(\mathrm{a}-\mathrm{c})$ concern chain length $n=100$ and $\varepsilon_{g} / \varepsilon_{a}$ ratio equals 0 (solid triangles), 1 (open circles), 2 (open triangles) and 4 (open squares). 
a

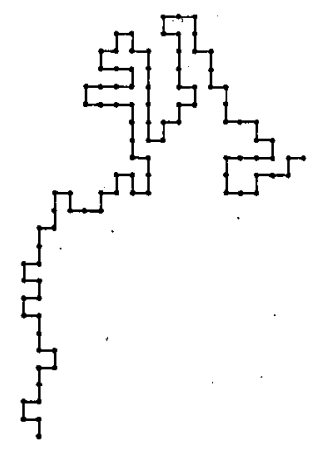

c

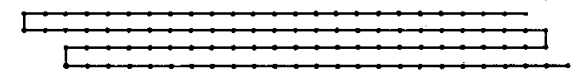

d

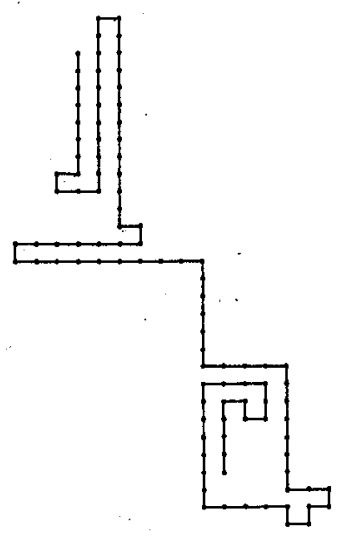

b

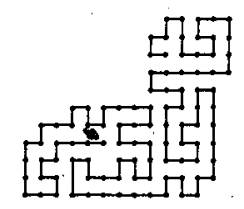

Fig. 4. Representative conformations of the model chain. The case of flexible chain $\left(\varepsilon_{g} / \varepsilon_{a}=0\right)$ for temperatures $T^{*}=1.6(\mathrm{a})$ and $0.6(\mathrm{~b})$. The case of stiff chain $\left(\varepsilon_{g} / \varepsilon_{a}=4\right)$ for temperatures $T^{*}=2.7$ (c) and $1.3(\mathrm{~d})$.

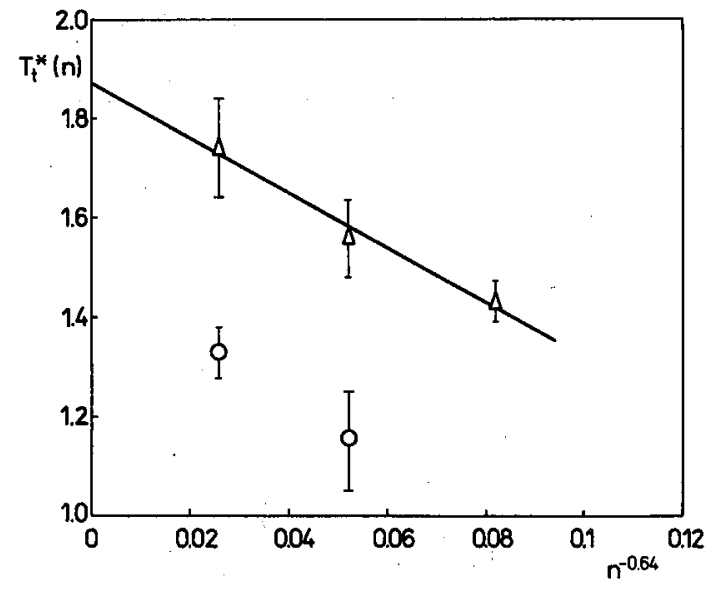

Fig. 5. The plot of the critical temperature $T_{t}^{*}$ vs. $n^{-0.64}$. The case of $\varepsilon_{g} / \varepsilon_{a}=2$ (open triangles) and 1 (open circles). 


\section{TABLE II}

The numerical values of $T_{t}^{*}(n)$ for various systems.

\begin{tabular}{r|c|l}
\hline \multicolumn{1}{c|}{$n$} & $\varepsilon_{g} / \varepsilon_{a}$ & \multicolumn{1}{c}{$T_{t}^{*}$} \\
\hline 20 & 0 & $0.82^{\mathrm{a}}$ \\
40 & 0 & $1.0^{\mathrm{a}}$ \\
50 & 2 & 1.43 \\
80 & 0 & $1.2^{\mathrm{a}}$ \\
100 & 0 & 1.14 \\
100 & 1 & 1.18 \\
100 & 2 & 1.56 \\
100 & 4 & 2.26 \\
160 & 0 & $1.18^{\mathrm{a}}$ \\
300 & 1 & 1.38 \\
300 & 2 & 1.74 \\
$\infty$ & 2 & 1.87 \\
$\mathrm{a}-$ Data extracted from [21]
\end{tabular}

\section{Conclusions}

The model systems studied in this work correspond to a polymer chain on a strongly adsorbing surface or in a thin film of solution [18-19]. We found in our Monte Carlo studies that the interplay between short range conformational stiffness of the polymer chain and repulsive-attractive interactions of chain segments may significantly change the temperature of the collapse transition of a polymer coil. With the increasing of the chain length the transition temperature.increases. It means that a weaker attractive force between polymer segments is needed for the onset of the collapse transition of a stiff chain than it is in the case of more flexible one. The transition at higher temperatures (for less flexible polymers) is more cooperative and a low temperature state exhibits the substantial local and global ordering of chain segments [14].

\section{References}

[1] P.J. Flory, Principles of Polymer Chemistry, Cornell University Press, Ithaca, New York 1953.

[2] P.G. de Gennes, Scaling Concepts in Polymer Physics, Cornell University Press, Ithaca, New York 1973.

[3] I.M. Lifshitz, A.Yu. Grosberg, A.R. Grosberg, Rev. Mod. Phys. 50, 683 (1978).

[1] K. Binder, in: Phase Transilions and Critical Phenomena, Vol. 8, eds. J.L. Lebowitz and C. Domb, Academic Press, New York 1983.

[5] J. Skolnick, A. Kolinski, Annu. Rev. Phys. Chem. 40, 207 (1989).

[6] J. Skolnick, A. Kolinski, R. Yaris, Biopolymers 26, 937 (1987). 
[7] A. Sikorski, J. Skolnick, Biopolymers 28, 1097 (1989).

[8] S. Redner, P.J. Reynolds, J. Phys. A: Math. Gen. 14, 2679 (1981).

[9] A.R. Khokhlov, Polymer 22, 447 (1981).

[10] A. Baumgartner, in: Applications of the Monte Carlo Method in Statistical Physics, Chap. 5, ed. K. Binder, Springer Verl., Berlin-Heidelberg-New York 1984.

[11] M.E. Fisher, B.J. Hiley, J. Chem, Phys. 34, 1253 (1961).

[12] K. Kremer, A. Baumgartner, K. Binder, J. Phys. A: Math. Gen. 15, 2879 (1981).

[13] A. Kolinski, J. Skolnick, R. Yaris, Macromolecules 20, 438 (1987).

[14] A. Kolinski,J. Skolnick, R. Yaris, J. Chem. Phys. 85, 3585 (1986).

[15] J.M. Stephen, J.L. McCauley, Phys. Lett. A 44, 89 (1973).

[16] J.M. Stephen, Phys. Lell. A 53, 263 (1975).

[17] B. Duplantier, Europhys. Letl. 1, 491 (1986).

[18] R. Vilanove, F. Rondelez, Phys. Rev. Lell. 45, 1502 (1980).

[19] R. Vilanove, D. Poupinet, F. Rondelez, Macromolecules 21, 2880 (1988).

[20] F.T. Wall, J. Mazur, Ann. N. Y. Acad. Sci. 89, 608 (1961).

[21] A. Baumgartner, J. Phys. 43, 1407 (1982).

[22] J. Tobochnik, I. Webman, J.L. Lebowitz, M.H. Kalos, Macromolecules 15,549 (1982).

[23] T.M. Birshtein, S.V. Buldyrev, A.M. Elyashevitch, Polymer 26, 1814. (1985).

[24] M. Bishop, J. Chem. Phys. 89, 1719 (1988).

[25] K. Binder, Monte Carlo Method in Statistical Physics, Springer Verl., Berlin-Heidelberg-New York 1979.

[26] I. Carmesin, K. Kremer, Macromolecules 21, 2819 (1988).

[27] N. Madras, A.D. Sokal, J. Stat. Phys. 47, 573 (1987). 